\title{
Genetic Variability, Heritability and Genetic Advance Analysis in Segregating Population of Black Gram [Vigna mungo (L.) Hepper]
}

\author{
Rakesh Gandi*, N. Shunmugavalli and Muthuswamy \\ Agricultural College and Research Institute, Killikulam (TNAU), India \\ *Corresponding author
}

\section{A B S T R A C T}

\begin{tabular}{|c|}
\hline Keywords \\
\hline $\begin{array}{l}\text { Black gram, } \\
\text { Variability, } \\
\text { Heritability and } \\
\text { Genetic advance as } \\
\text { per cent of Mean }\end{array}$ \\
\hline Article Info \\
\hline $\begin{array}{l}\text { Accepted: } \\
07 \text { January } 2018 \\
\text { Available Online: } \\
10 \text { February } 2018\end{array}$ \\
\hline
\end{tabular}

\section{Introduction}

Black gram is also called as urd bean and black lentil. It is an important multipurpose grain legume extensively cultivated in arid, semi-arid and subtropics. Black gram is photo insensitive in nature and can be cultivated throughout the year. Black gram fits well in different cropping system as it is relatively drought tolerant. It is also grown as catch crop, mulch crop inter crop, mixed crop and green crop. Jayamani et al., (2012) reported that about 70 percent of the total pulse production area is in the central and southern parts of the country and this contributes about more than 77 per cent of the total production.

\begin{abstract}
In the present investigation, nine crosses of black gram (Vigna mungo L.) were evaluated in $\mathrm{F}_{2}$ and $\mathrm{F}_{3}$ generations for the effective selection for yield and yield components. The segregating generation of the crosses included were PU 06-20 x VBN 4, IPU 2006-01 x ADT3, IPU 2006-01 x VBN 4, IPU 2006-01 x TNY LOCAL, IPU 02-33 x VBN 4, IPU 02-33 x VBN 4, IPU 02-33 x TNY LOCAL, WBG 26 x VBN 3, MASH 114 x VBN 3 and MASH $114 \times$ TNY LOCAL. The estimates of PCV values are higher than that of GCV. The genotypic variance was found to be high for clusters per plant, pods per cluster and single plant yield in both the generations in the cross MASH $114 \mathrm{x}$ TNY LOCAL. High heritability coupled with high genetic advance as per cent of mean in both generations was cluster by shortlisted as the ones amenable for selection.
\end{abstract}


extent of variation which would help in assessing the variability and factors for limited progress made in blackgram. From this point of view, the present investigation was undertaken involving nine cross combinations of black gram genotypes.

\section{Materials and Methods}

The material for the present study was generated at the Department of Plant Breeding and Genetics, Agricultural College and Research Institute (TNAU) Killikulam. Nine high yielding cross combinations were selected from the $F_{1}$ generation of the previous study based single plant yield along with the VBN 4 variety as the check. The experiment was laid out in homogenous block following randomized block design (RBD) with three replications. The spacing between the rows was $30 \mathrm{~cm}$ and spacing between the individual plants was $10 \mathrm{~cm}$. Recommended agronomic practices were followed to raise the crop. Observations on 8 quantitative characters were recorded. The estimates of variability, heritability and genetic advance were estimated by using the statistical methods suggested by Burton (1952), Lush (1944) and Johnson et al., (1955) respectively.

\section{Results and Discussion}

A critical estimate of genetic variability is a pre - requisite for initiating appropriate breeding procedures in crop improvement programme, since many characters of economic importance are highly influenced by environmental conditions. The improvement of mainly depends upon the amount, nature and magnitude of the variability present in the population (Mothilal, 2000). The genotypic variance was found to be high for yield contributing characters like clusters per plant, pods per cluster and single plant yield in both the generations of the cross MASH $114 \mathrm{x}$ TNY LOCAL (Table 1). For pods per plant, the genotypic variance was high in cross IPU 02-33 x TNY LOCAL in both the generation. In $F_{3}$, the same combination recorded high genotypic variance for plant height, pods per cluster and seeds per pod. The estimates of phenotypic coefficient of variation (PCV) were higher than genotypic coefficient of variation $(\mathrm{GCV})$. The difference was comparatively moderate inferring that there was environmental influence in all the traits studied. This is in the agreement to the findings of Joel and Thangavelu (1997). In the $\mathrm{F}_{2}$ and $\mathrm{F}_{3}$ generations of the cross MASH 114 $\mathrm{x}$ VBN 3, the traits pods per plant, pods per cluster, pod length and single plant yield recorded high GCV and PCV, while, pods per plant registered low PCV and GCV values. The GCV was found to be low in $\mathrm{F}_{2}$ and $\mathrm{F}_{3}$ of the cross IPU 02-33 x TNY LOCAL. The $\mathrm{PCV}$ and GCV were low in $\mathrm{F}_{3}$ generation of IPU 02-33 x VBN 4 for single plant yield. These results are in conformity with the earlier findings of Konda et al., (2009) in black gram indicating the better scope for improvement of pods per plant and single plant yield of the materials under present study. High variability was observed for clusters per plant in MASH $114 \mathrm{x}$ TNY LOCAL $\left(\mathrm{F}_{2}\right.$ and $\left.\mathrm{F}_{3}\right)$, IPU 2006-01 $x$ TNY LOCAL $\left(\mathrm{F}_{2}\right.$ and $\left.\mathrm{F}_{3}\right)$ and PU 06-20 x VBN $4\left(\mathrm{~F}_{3}\right)$ indicated that better scope of selection for this trait. But for the rest of the crosses in both the segregating generations, low values were recorded for GCV and PCV, thus indicating low variability for this trait. Similar reports were given by Singh et al., (2009) in black gram (Table 1). Low variability for 100 seed weight was noticed in all crosses in both $F_{2}$ and $F_{3}$. This result was similar to the previous reports given by Meshram et al., (2013) in black gram. Majority of the combinations evaluated, showed high GCV and PCV for single plant yield and pods per cluster in both $F_{2}$ and $F_{3}$ generations and these findings are in conformity with the earlier reports of Meshram et al., (2013). 
Table.1 Variability for different characters in blackgram

\begin{tabular}{|c|c|c|c|c|c|c|c|c|c|c|c|c|c|}
\hline \multirow[t]{2}{*}{ Characters } & & \multicolumn{4}{|c|}{ PU 06-20 x VBN 4} & \multicolumn{4}{|c|}{ IPU 2006-01 x ADT 3} & \multicolumn{4}{|c|}{ IPU 2006- 01 x VBN 4} \\
\hline & & PV & GV & PCV & GCV & PV & GV & PCV & GCV & PV & GV & PCV & GCV \\
\hline \multirow{2}{*}{$\begin{array}{l}\text { Plant } \\
\text { height }\end{array}$} & $\mathrm{F}_{2}$ & 12.81 & 15.77 & 21.49 & 18.77 & 1.85 & 0.16 & 7.25 & 2.19 & 3.15 & 1.492 & 8.02 & 5.52 \\
\hline & $\mathrm{F}_{3}$ & 15.11 & 13.41 & 16.92 & 17.96 & 2.18 & 0.15 & 7.89 & 2.10 & 3.22 & 2.26 & 7.59 & 6.36 \\
\hline \multirow{2}{*}{$\begin{array}{l}\text { Clusters } \\
\text { per plant }\end{array}$} & $\mathrm{F}_{2}$ & 1.00 & 0.30 & 12.86 & 7.01 & 0.28 & 0.05 & 8.40 & 3.76 & 0.40 & 0.08 & 8.30 & 3.85 \\
\hline & $\mathrm{F}_{3}$ & 6.84 & 5.30 & 33.32 & 29.32 & 0.06 & 0.06 & 3.83 & 3.80 & 0.51 & 0.11 & 8.36 & 3.89 \\
\hline \multirow{2}{*}{$\begin{array}{l}\text { Pods per } \\
\text { cluster }\end{array}$} & $\mathrm{F}_{2}$ & 2.84 & 1.53 & 31.23 & 26.66 & 0.59 & 0.26 & 28.83 & 24.07 & 0.25 & 0.11 & 28.04 & 23.83 \\
\hline & $\mathrm{F}_{3}$ & 2.42 & 0.67 & 42.97 & 22.61 & 0.48 & 0.20 & 21.46 & 21.35 & 0.32 & 0.15 & 29.06 & 24.85 \\
\hline \multirow[t]{2}{*}{ Pod length } & $\mathrm{F}_{2}$ & 0.69 & 0.39 & 17.59 & 13.32 & 0.82 & 0.28 & 17.88 & 10.52 & 0.81 & 0.46 & 19.05 & 14.32 \\
\hline & $\mathrm{F}_{3}$ & 0.31 & 0.17 & 16.03 & 11.86 & 0.33 & 0.33 & 10.33 & 10.34 & 0.78 & 0.49 & 19.36 & 15.36 \\
\hline \multirow{2}{*}{$\begin{array}{l}\text { Pods per } \\
\text { plant }\end{array}$} & $\mathrm{F}_{2}$ & 40.23 & 29.31 & 66.26 & 48.65 & 2.12 & 1.48 & 18.47 & 12.35 & 5.88 & 4.24 & 19.41 & 13.20 \\
\hline & $\mathrm{F}_{3}$ & 115.30 & 31.92 & 42.97 & 22.61 & 2.08 & 2.05 & 19.06 & 12.38 & 6.67 & 4.88 & 19.56 & 13.69 \\
\hline \multirow{2}{*}{$\begin{array}{l}\text { Seeds per } \\
\text { pod }\end{array}$} & $\mathrm{F}_{2}$ & 0.73 & 0.24 & 17.74 & 10.34 & 0.76 & 0.21 & 17.15 & 9.09 & 0.64 & 0.40 & 16.89 & 13.36 \\
\hline & $\mathrm{F}_{3}$ & 0.48 & 0.29 & 14.38 & 11.32 & 1.11 & 0.31 & 17.89 & 9.56 & 0.77 & 0.54 & 17.56 & 14.69 \\
\hline \multirow{2}{*}{$\begin{array}{l}100 \text { seed } \\
\text { weight }\end{array}$} & $\mathrm{F}_{2}$ & 0.30 & 0.05 & 5.41 & 4.67 & 0.19 & 0.14 & 8.99 & 7.71 & 0.03 & 0.02 & 6.97 & 5.52 \\
\hline & $\mathrm{F}_{3}$ & 0.32 & 0.21 & 11.48 & 9.39 & 0.18 & 0.14 & 8.82 & 7.76 & 0.06 & 0.05 & 7.02 & 6.65 \\
\hline \multirow{2}{*}{$\begin{array}{l}\text { Single } \\
\text { plant yield }\end{array}$} & $\mathrm{F}_{2}$ & 3.40 & 1.40 & 35.78 & 37.57 & 2.40 & 1.78 & 45.16 & 52.35 & 0.95 & 0.88 & 33.56 & 34.88 \\
\hline & $\mathrm{F}_{3}$ & 3.57 & 2.66 & 48.15 & 55.77 & 2.60 & 1.86 & 45.18 & 53.45 & 1.01 & 0.94 & 33.65 & 34.92 \\
\hline
\end{tabular}

Table.1 Conti....

\begin{tabular}{|c|c|c|c|c|c|c|c|c|c|c|c|c|c|}
\hline \multirow[t]{2}{*}{ Characters } & & \multicolumn{4}{|c|}{ IPU 2006-01 x TNY LOCAL } & \multicolumn{4}{|c|}{ IPU 02-33 x VBN 4} & \multicolumn{4}{|c|}{ IPU 02-33 xTNY LOCAL } \\
\hline & & PV & GV & PCV & GCV & PV & GV & PCV & GCV & PV & GV & PCV & GCV \\
\hline \multirow[t]{2}{*}{ Plant height } & $\mathrm{F}_{2}$ & 14.36 & 8.17 & 15.96 & 12.04 & 14.36 & 8.17 & 15.96 & 12.04 & 12.21 & 9.30 & 20.56 & 17.94 \\
\hline & $\mathrm{F}_{3}$ & 3.49 & 8.70 & 8.21 & 12.95 & 3.49 & 8.70 & 8.21 & 12.95 & 22.58 & 16.77 & 25.48 & 21.96 \\
\hline \multirow{2}{*}{$\begin{array}{l}\text { Clusters per } \\
\text { plant }\end{array}$} & $\mathrm{F}_{2}$ & 1.28 & 0.58 & 15.77 & 10.58 & 0.49 & 0.29 & 9.81 & 7.51 & 0.88 & 0.44 & 10.88 & 7.73 \\
\hline & $\mathrm{F}_{3}$ & 3.89 & 2.95 & 27.22 & 23.70 & 2.14 & 0.33 & 16.92 & 6.65 & 1.80 & 0.54 & 15.51 & 8.50 \\
\hline \multirow{2}{*}{$\begin{array}{l}\text { Pods per } \\
\text { cluster }\end{array}$} & $\mathrm{F}_{2}$ & 0.20 & 0.09 & 28.61 & 24.05 & 0.30 & 0.29 & 47.33 & 42.88 & 1.46 & 1.07 & 28.04 & 23.83 \\
\hline & $\mathrm{F}_{3}$ & 5.73 & 7.64 & 22.27 & 26.28 & 4.97 & 3.73 & 48.39 & 43.89 & 12.66 & 6.59 & 26.28 & 22.27 \\
\hline \multirow[t]{2}{*}{ Pod length } & $\mathrm{F}_{2}$ & 0.69 & 0.42 & 17.55 & 13.74 & 2.57 & 2.42 & 33.78 & 33.17 & 0.92 & 0.42 & 20.22 & 13.66 \\
\hline & $\mathrm{F}_{3}$ & 0.62 & 0.17 & 16.68 & 8.80 & 0.73 & 0.43 & 17.83 & 13.67 & 0.63 & 0.37 & 16.73 & 12.76 \\
\hline \multirow{2}{*}{$\begin{array}{l}\text { Pods per } \\
\text { plant }\end{array}$} & $\mathrm{F}_{2}$ & 8.92 & 6.30 & 17.79 & 12.01 & 29.21 & 123.98 & 19.24 & 18.88 & 8.41 & 6.09 & 44.56 & 38.05 \\
\hline & $\mathrm{F}_{3}$ & 6.62 & 9.22 & 49.70 & 57.36 & 31.23 & 25.69 & 57.36 & 49.70 & 12.69 & 9.11 & 72.32 & 52.2 \\
\hline \multirow{2}{*}{$\begin{array}{l}\text { Seeds per } \\
\text { pod }\end{array}$} & $\mathrm{F}_{2}$ & 0.75 & 0.46 & 17.55 & 13.74 & 2.91 & 2.81 & 33.78 & 33.17 & 0.91 & 0.41 & 20.22 & 13.66 \\
\hline & $\mathrm{F}_{3}$ & 0.63 & 1.11 & 13.26 & 17.53 & 0.70 & 0.53 & 14.57 & 12.70 & 17.96 & 9.36 & 72.32 & 52.21 \\
\hline \multirow{2}{*}{$\begin{array}{l}100 \text { seed } \\
\text { weight }\end{array}$} & $\mathrm{F}_{2}$ & 0.05 & 0.05 & 4.69 & 4.56 & 0.58 & 0.53 & 15.51 & 14.83 & 0.15 & 0.09 & 8.18 & 6.15 \\
\hline & $\mathrm{F}_{3}$ & 0.11 & 0.24 & 7.02 & 9.95 & 0.63 & 0.62 & 16.02 & 15.90 & 0.05 & 0.05 & 4.83 & 4.74 \\
\hline
\end{tabular}




\begin{tabular}{|c|c|c|c|c|c|c|c|c|c|c|c|c|c|}
\hline \multirow[t]{2}{*}{ Characters } & & \multicolumn{4}{|c|}{ WBG $26 \times$ VBN 3} & \multicolumn{4}{|c|}{ MASH 114 xVBN 3} & \multicolumn{4}{|c|}{ MASH 114 xTNY LOCAL } \\
\hline & & PV & GV & PCV & GCV & PV & GV & PCV & GCV & PV & GV & PCV & GCV \\
\hline \multirow[t]{2}{*}{ Plant height } & $\mathrm{F}_{2}$ & 12.21 & 9.30 & 20.56 & $\begin{array}{l}17.94 \\
21.06\end{array}$ & 4.79 & 2.48 & 9.57 & 6.89 & 6.31 & 5.330 & 13.60 & 12.50 \\
\hline & $\mathrm{F}_{3}$ & 22.58 & 16.77 & 25.48 & & 7.92 & 5.13 & 11.77 & 9.47 & 6.17 & 3.292 & 12.90 & 9.42 \\
\hline \multirow{2}{*}{$\begin{array}{l}\text { Clusters per } \\
\text { plant }\end{array}$} & $\mathrm{F}_{2}$ & 0.69 & 0.25 & 13.79 & 8.29 & 1.22 & 0.44 & 13.79 & 8.29 & 3.41 & 2.22 & 31.41 & 25.36 \\
\hline & $\mathrm{F}_{3}$ & 0.68 & 0.44 & 10.51 & 8.50 & 1.23 & 0.47 & 13.86 & 8.59 & 4.81 & 3.16 & 32.89 & 26.65 \\
\hline \multirow{2}{*}{$\begin{array}{l}\text { Pods per } \\
\text { cluster }\end{array}$} & $\mathrm{F}_{2}$ & 2.24 & 1.24 & 9.36 & 22.99 & 0.11 & 0.03 & 7.42 & 3.48 & 2.41 & 1.34 & 28.04 & 23.83 \\
\hline & $\mathrm{F}_{3}$ & 3.39 & 1.77 & 31.55 & 27.68 & 0.29 & 0.02 & 8.68 & 3.98 & 3.05 & 1.75 & 28.98 & 24.82 \\
\hline \multirow[t]{2}{*}{ Pod length } & $\mathrm{F}_{2}$ & 0.89 & 0.38 & 20.01 & 13.16 & 0.79 & 0.29 & 18.84 & 11.59 & 0.90 & 0.39 & 20.01 & 13.16 \\
\hline & $\mathrm{F}_{3}$ & 0.69 & 0.40 & 16.73 & 12.76 & 0.78 & 0.32 & 18.98 & 12.24 & 1.65 & 0.66 & 21.56 & 13.69 \\
\hline \multirow{2}{*}{$\begin{array}{l}\text { Pods per } \\
\text { plant }\end{array}$} & $\mathrm{F}_{2}$ & 0.12 & 0.34 & 56.44 & 1.18 & 0.61 & 0.41 & 13.77 & 0.76 & 4.23 & 2.05 & 56.44 & 5.86 \\
\hline & $F_{3}$ & 12.31 & 3.51 & 72.30 & 16.00 & 0.25 & 0.50 & 14.02 & 1.21 & 1.75 & 1.32 & 58.03 & 3.05 \\
\hline \multirow{2}{*}{$\begin{array}{l}\text { Seeds per } \\
\text { pod }\end{array}$} & $\mathrm{F}_{2}$ & 0.86 & 0.37 & 20.01 & 13.16 & 0.89 & 0.33 & 18.84 & 11.56 & 0.95 & 0.41 & 20.01 & 13.16 \\
\hline & $\mathrm{F}_{3}$ & 1.05 & 0.24 & 18.49 & 8.82 & 1.25 & 0.53 & 19.36 & 12.58 & 0.55 & 0.29 & 20.09 & 14.65 \\
\hline \multirow{2}{*}{$\begin{array}{l}100 \text { seed } \\
\text { weight }\end{array}$} & $\mathrm{F}_{2}$ & 0.13 & 0.05 & 7.67 & 4.90 & 0.15 & 0.07 & 8.03 & 5.52 & 0.14 & 0.05 & 7.67 & 0.37 \\
\hline & $\mathrm{F}_{3}$ & 0.05 & 0.04 & 4.83 & 4.74 & 0.15 & 0.07 & 8.06 & 5.65 & 0.13 & 0.05 & 7.78 & 0.36 \\
\hline \multirow{2}{*}{$\begin{array}{l}\text { Single plant } \\
\text { yield }\end{array}$} & $\mathrm{F}_{2}$ & 1.60 & 1.35 & 31.84 & 34.72 & 1.65 & 1.39 & 31.80 & 34.72 & 2.00 & 1.53 & 33.98 & 38.87 \\
\hline & $\mathrm{F}_{3}$ & 1.94 & 1.92 & 39.11 & 39.27 & 1.62 & 1.45 & 32.76 & 34.67 & 2.05 & 1.55 & 33.54 & 38.56 \\
\hline
\end{tabular}

Table.2 Hertiability and GA as per cent mean for different characters in black gram

\begin{tabular}{|c|c|c|c|c|c|c|}
\hline \multirow{2}{*}{ Characters } & \multicolumn{2}{|c|}{ PU 06-20 x VBN 4} & \multicolumn{2}{|c|}{ IPU 2006-01 x ADT 3} & \multicolumn{2}{|c|}{ IPU 2006- 01 x VBN 4} \\
\hline & Heritability & GA as \% Mean & Heritability & GA as \% Mean & Heritability & GA as \% Mean \\
\hline \multirow[t]{2}{*}{ Plant height } & 76.11 & 33.77 & 59.67 & 1.36 & 47.13 & 7.82 \\
\hline & 88.34 & 32.85 & 69.46 & 1.68 & 68.29 & 8.68 \\
\hline \multirow{2}{*}{$\begin{array}{l}\text { Clusters per } \\
\text { plant }\end{array}$} & 29.72 & 7.87 & 20.65 & 3.48 & 21.43 & 3.68 \\
\hline & 77.64 & 53.15 & 24.36 & 3.75 & 32.36 & 4.42 \\
\hline \multirow{2}{*}{$\begin{array}{l}\text { Pods per } \\
\text { cluster }\end{array}$} & 72.22 & 46.88 & 69.48 & 41.40 & 72.32 & 41.71 \\
\hline & 27.65 & 24.50 & 72.25 & 42.54 & 75.89 & 43.43 \\
\hline \multirow[t]{2}{*}{ Pod length } & 57.32 & 20.79 & 34.36 & 12.75 & 56.25 & 22.17 \\
\hline & 54.46 & 18.09 & 36.24 & 12.86 & 63.65 & 23.36 \\
\hline \multirow[t]{2}{*}{ Pods perplant } & 53.12 & 73.60 & 44.23 & 17.02 & 46.84 & 18.49 \\
\hline & 27.63 & 24.50 & 66.72 & 15.36 & 49.25 & 18.89 \\
\hline \multirow[t]{2}{*}{ Seeds per pod } & 34.12 & 12.42 & 28.46 & 9.92 & 62.36 & 21.79 \\
\hline & 61.25 & 18.36 & 33.19 & 9.87 & 69.47 & 22.86 \\
\hline \multirow{2}{*}{$\begin{array}{l}100 \text { seed } \\
\text { weight }\end{array}$} & 74.26 & 8.31 & 73.25 & 13.62 & 62.24 & 9.01 \\
\hline & 67.37 & 15.84 & 76.89 & 14.87 & 64.56 & 10.03 \\
\hline $\begin{array}{l}\text { Single plant } \\
\text { yield }\end{array}$ & $\begin{array}{l}90.65 \\
74.28\end{array}$ & $\begin{array}{l}70.18 \\
85.62\end{array}$ & $\begin{array}{l}74.65 \\
76.04\end{array}$ & $\begin{array}{l}80.27 \\
81.69\end{array}$ & $\begin{array}{l}92.25 \\
93.26\end{array}$ & $\begin{array}{l}66.57 \\
66.59\end{array}$ \\
\hline
\end{tabular}




\begin{tabular}{|c|c|c|c|c|c|c|}
\hline \multirow[t]{2}{*}{ Characters } & \multicolumn{2}{|c|}{ IPU 2006-01 x TNY LOCAL } & \multicolumn{2}{|c|}{ IPU02-33 x VBN 4} & \multicolumn{2}{|c|}{ IPU 02-33 x TNY LOCAL } \\
\hline & Heritability & GA as $\%$ Mean & Heritability & GA as $\%$ Mean & Heritability & GA as $\%$ Mean \\
\hline \multirow[t]{2}{*}{ Plant height } & 56.23 & 18.72 & 56.45 & 9.53 & 84.60 & 60.18 \\
\hline & 62.76 & 19.56 & 58.24 & 9.67 & 99.21 & 8.02 \\
\hline \multirow{2}{*}{$\begin{array}{l}\text { Clusters per } \\
\text { plant }\end{array}$} & 45.02 & 14.63 & 84.12 & 58.55 & 50.23 & 11.31 \\
\hline & 75.23 & 42.50 & 15.65 & 5.45 & 30.65 & 9.59 \\
\hline \multirow{2}{*}{$\begin{array}{l}\text { Pods per } \\
\text { cluster }\end{array}$} & 70.12 & 41.66 & 82.25 & 80.03 & 72.24 & 41.71 \\
\hline & 71.19 & 38.89 & 84.26 & 80.09 & 71.47 & 38.89 \\
\hline \multirow[t]{2}{*}{ Pod length } & 61.12 & 22.16 & 96.47 & 67.09 & 45.65 & 19.03 \\
\hline & 27.23 & 9.57 & 58.23 & 21.61 & 58.25 & 20.06 \\
\hline \multirow{2}{*}{$\begin{array}{l}\text { Pods per } \\
\text { plant }\end{array}$} & 45.25 & 16.71 & 96.26 & 38.19 & 72.56 & 66.93 \\
\hline & 74.23 & 88.70 & 75.28 & 86.70 & 52.48 & 77.66 \\
\hline \multirow[t]{2}{*}{ Seeds per pod } & 61.69 & 22.16 & 96.52 & 67.05 & 45.42 & 19.03 \\
\hline & 57.26 & 20.66 & 64.48 & 24.65 & 52.68 & 77.66 \\
\hline \multirow{2}{*}{$\begin{array}{l}100 \text { seed } \\
\text { weight }\end{array}$} & 94.89 & 9.130 & 45.42 & 19.03 & 56.61 & 9.53 \\
\hline & 49.27 & 10.20 & 52.68 & 77.66 & 96.23 & 9.58 \\
\hline \multirow{2}{*}{$\begin{array}{l}\text { Single plant } \\
\text { yield }\end{array}$} & 66.29 & 60.18 & 91.24 & 29.21 & 56.45 & 9.53 \\
\hline & 90.01 & 63.97 & 49.23 & 10.20 & 58.24 & 9.67 \\
\hline
\end{tabular}

\begin{tabular}{|c|c|c|c|c|c|c|}
\hline \multirow[t]{2}{*}{ Characters } & \multicolumn{2}{|c|}{ WBG 26 x VBN 3} & \multicolumn{2}{|c|}{ MASH 114 xVBN 3} & \multicolumn{2}{|c|}{ MASH 114 x TNY LOCAL } \\
\hline & Heritability & $\begin{array}{c}\text { GA as \% } \\
\text { Mean }\end{array}$ & Heritability & $\begin{array}{c}\text { GA as \% } \\
\text { Mean }\end{array}$ & Heritability & $\begin{array}{c}\text { GA as \% } \\
\text { Mean }\end{array}$ \\
\hline \multicolumn{7}{|l|}{ Plant height } \\
\hline \multirow{2}{*}{$\begin{array}{l}\text { Clusters per } \\
\text { plant }\end{array}$} & 36.65 & 10.27 & 36.81 & 10.28 & 65.45 & 42.20 \\
\hline & 42.25 & 11.89 & 39.26 & 11.05 & 69.12 & 43.36 \\
\hline \multirow{2}{*}{$\begin{array}{l}\text { Pods per } \\
\text { cluster }\end{array}$} & 63.23 & 14.75 & 69.23 & 11.61 & 72.26 & 41.71 \\
\hline & 76.12 & 49.83 & 67.69 & 12.62 & 76.61 & 43.98 \\
\hline \multirow[t]{2}{*}{ Pod length } & 43.44 & 17.84 & 37.72 & 14.69 & 43.46 & 17.84 \\
\hline & 58.68 & 20.06 & 43.23 & 14.70 & 45.24 & 17.86 \\
\hline \multirow[t]{2}{*}{ Pods per plant } & 55.43 & 64.54 & 44.46 & 28.06 & 55.05 & 64.54 \\
\hline & 52.56 & 77.66 & 49.19 & 26.89 & 61.09 & 66.39 \\
\hline \multirow[t]{2}{*}{ Seeds per pod } & 43.24 & 17.84 & 37.23 & 14.69 & 43.23 & 17.84 \\
\hline & 22.44 & 8.68 & 44.45 & 15.70 & 46.25 & 18.97 \\
\hline \multirow{2}{*}{$\begin{array}{l}100 \text { seed } \\
\text { weight }\end{array}$} & 47.54 & 6.34 & 47.23 & 7.841 & 48.32 & 6.44 \\
\hline & 96.16 & 9.58 & 45.44 & 7.89 & 49.68 & 64.49 \\
\hline \multirow{2}{*}{$\begin{array}{l}\text { Single plant } \\
\text { yield }\end{array}$} & 84.60 & 60.18 & 84.44 & 60.17 & 0.88 & 64.69 \\
\hline & 99.21 & 8.02 & 82.61 & 60.02 & 0.79 & 62.63 \\
\hline
\end{tabular}


Genetic coefficient of variability along with the heritability gives an idea of expected genetic gain from selection (Burton, 1952). The heritability estimates in the present investigation are of broad sense containing additive and non-additive portions of genetic variance and hence cannot provide clear predictability of breeding value of genotype. As such, heritability value can be reliable measure of predicting the resultants effects of selection only it is when accompanied by high genetic advance expressed as percentage of mean (Johnson et al., 1955).

High heritability along with high genetic advance as per cent of mean for single plant yield in all crosses indicated the presence of additive gene effects (Table 2). This was in conformity with the earlier reports of Anbuselvam et al., (2010).

High heritability along with high genetic advance as per cent of mean was exhibited by IPU 02-33 $x$ TNY LOCAL in both generations for the characters clusters per plant, pods per cluster, pods per plant, seeds per pod and single plant yield indicating the predominance of additive gene effects and selection based on these characters is effective. In the present investigation, high heritability coupled with moderate genetic advance recorded for pods per plant in crosses IPU 02-33 x TNY LOCAL $\left(\mathrm{F}_{2}\right)$ and MASH $114 \times$ VBN $3\left(\mathrm{~F}_{2}\right.$ and $\left.\mathrm{F}_{3}\right)$. For the character seeds per pod was also had high heritability with moderate genetic advance as per cent in cross PU 06-20 x VBN $4\left(\mathrm{~F}_{3}\right)$. Therefore by restoring to straight selection based on these characters, further improvement can be achieved reported by Gupta and Gupta (1977). Moderate heritability with high genetic advance was recorded by cross IPU 2006-01 x TNY LOCAL $\left(\mathrm{F}_{3}\right)$ for seeds per pod indicating predominant role for nonadditive factors. Similar results reported earlier by Priyanka et al., (2011).
Moderate with low genetic advance was observed for seeds per pod in the $\mathrm{F}_{3}$ of the cross IPU 2006-01 x ADT3, for hundred seed weight in the cross MASH 114 x VBN 3 and for single plant yield in both generations of IPU 02-33 x TNY LOCAL indicating the predominant role of non-additive factors in the expression of these characters. This confirms the earlier findings of Pandey et al., (2007). Low heritability coupled with low genetic advance recorded by pod length for cross IPU 2006-01 x TNY LOCAL $\left(\mathrm{F}_{3}\right)$ indicates the non-additive gene action which is not amenable for selection of this cross based on this trait confirming the earlier findings of Srivastava and Singh (2012) in green gram.

\section{References}

Anbu Selvam, R. Elangaimannan, M. Venkatesan, P. Karthikeyan and K. Palaniraja. 2010. Chemically induced mutagenesis in Blackgram (Vigna mungo (L.) Hepper). Electronic Journal of Plant Breeding, 1(4): 921-924.

Burton, G.W. 1952. Quantitative inheritance in grasses. Proc. $6^{\text {th }}$ Int. Grassland Congress, 1: 277-283.

Gupta, V.K. and Y.K. Gupta. 1997. Variability, inter relationships and path co-efficient analysis for some quantitative characters in sesame (Sesamum indicum L.). Indian $J$. Heridity., 9: 31-37.

Jain, H. K.1975. Breeding for yield and other attributes in grain legumes. Indian Journal of Genetics, 35: 169-187.

Jayamani, P., N. Kumaravadivel, N. Nadarajan, A.R. Muthiah, C. Durairaj, A. Kamalakannan, S. Pazhanivelan and K. Thiyagarajan. 2012. TNAU Black gram CO6: A High Yielding Short Duration Variety. Madras Agric. J., 99(1-3): 34-36.

Joel, J.A and S. Thangavelu. 1997. 
Variability, heritability and genetic advance in sesame. Madras Agric. J., 47:314-318.

Johnson, H.W., H.F. Robinson and R.E. Comstock. 1955. Estimates of genetic advance and environmental variability in soya bean. Agron. J., 47: 314-318.

Konda, C.R., P.H. Salimath and M.N. Mishra. 2009. Genetic variability studies in productivity and its components in black gram (Vigna mungo (L.) Hepper). Legume Res., 31(1): 59-61.

Meshram, M.P., R.I. Ali, A.N. Patil and M. Sunita. 2013. Variability studies in $\mathbf{M}_{3}$ Generation in blackgram (Vigna mungo (L.) Hepper). The Bioscan., 8(4): 13571361.

Mothilal, A. 2000.Genetic variability and pattern of segregation in $\mathrm{F}_{2}$ populations of sesame. (Sesamum indicum L.). J. Oilseeds Res., 17(2): 216 - 225.

Pandey, M.K., N. Srivastava and C.R. Kole.
2007. Selection strategy for augmentation of seed yield in mung bean (Vigna radiata (L.) Wilczek). Legume Res., 30(4): 243- 249.

Priyanka, B., D.P. Singh and R.K. Khulbe. 2011. Genetic variability and association analysis of advanced lines and cultivars following intervarietal and interspecific crosses in black gram. Journal of Crop Improvement, 38(1):67-70.

Ramanujam, S.1975. Genetic diversity, Stability and plant type in pulse crops. In: Proceedings of International Workshop Grain Legume, ICRISAT, Hyderabad, pp. 167-176.

Singh, T., A. Sharma and F.A. Alie.2009. Impact of environment on heritability and genetic gain for yield and its component traits in mungbean. Legume Res., 32(1): 55-58.

\section{How to cite this article:}

Rakesh, Gandi, N. Shunmugavalli and Muthuswamy. 2018. Genetic Variability, Heritability and Genetic Advance Analysis in Segregating Population of Black Gram (Vigna mungo (L.) Hepper). Int.J.Curr.Microbiol.App.Sci. 7(02): 703-709. doi: https://doi.org/10.20546/ijcmas.2018.702.088 